\author{
MARGIT EBERHARTER-AKSU \\ Uniwersytet Gdański \\ margit.eberharter-aksu@ug.edu.pl \\ ORCID: 0000-0001-7093-0500
}

\title{
JOLANTA HINC
}

Uniwersytet Gdański

jolanta.hinc@ug.edu.pl

ORCID: 0000-0003-2119-6578

\section{Übersetzungsstrategien von Grimms Rotkäppchen am Beispiel polnischer Übersetzungen}

\section{Translation strategies of Grimm's Little Red Riding Hood on the example of Polish translations}

\begin{abstract}
The fairy tales of the Brothers Grimm belong to the best-known literary works of Germany, besides the Luther Bible. They are currently published in 160 languages and have been part of the World Documentary Heritage since June 2005. In Poland, they are known in countless translations, adaptations and retellings, some of which have been greatly modified and adapted for teaching purposes and others are literally based on the original texts. The empirical part of the article refers to an experiment in which 19 subjects were presented with a translation task: to transfer selected passages of Little Red Riding Hood into Polish. In the runup to the study, it was hypothesized that the information about the status of the work as part of the World Document Heritage given to study participants should have an effect on the literal translation. The results of the experiment do not confirm the assumed hypothesis, but clearly show that the translation variants above all have to be seen as a continuation of the individual's experiences with the fairy tale, which strongly influences that individual's translation.
\end{abstract}

KEYWORDS: Grimm's fairytales, translation strategies, translation into Polish.

SCHLÜSSELWORTE: Grimms Märchen, Übersetzungsstrategien, Übersetzung ins Polnische. 


\section{GRIMMSCHE MÄRCHEN IN POLNISCHEN ÜBERSETZUNGEN}

In Polen wurden die grimmschen Märchen erst relativ spät und zumeist in Adaptionen populär. Zudem sind nur wenige der über 200 Märchen der Brüder Grimm in polnischen Übersetzungen wirklich bekannt. Während die Märchen in Westeuropa bis zum Jahre 1830 schon eine recht breite Bekanntheit erlangten, erschien in Polen erst im Jahr 1895 eine erste Auswahl von 16 Märchen (vgl. Halub 1986: 217). Dieses relativ späte Interesse überrascht in gewisser Hinsicht, als die Sammlung der Brüder Grimm im Zusammenhang mit der polnischen Romantik (1795-1864), die sich dem volkskundlichen Sammeln von Texten verschrieben hatte, enge Parallelen aufweist. Die Gründe für die zurückhaltende Rezeption liegen aber aller Wahrscheinlichkeit nach in der polenfeindlichen Politik Preußens und der österreichischen Teilungsmacht, wenngleich Jakob Grimm als Kritiker der Politik der Großmächte auftrat, sodass eine Ablehnung deutscher Einflüsse nachvollziehbar ist. In der folgenden positivistischen Strömung (1864-1891), die sich in Polen als Reaktion auf die Teilung der Nation und die verlorenen Freiheitskämpfe verbreitete, galten dann Märchen und romantische Vergangenheitsforschung als überholt und wurden abgelehnt. Aus diesen Gründen wurde erst 1896, zur Zeit der Neuromantik und des Jungen Polen, die erste polnische Gesamtausgabe der grimmschen Märchen mit dem Titel Bajki domowe i dziecinne (Haus- und Kindermärchen) verlegt. Sie wurde von der Ethnografin Zofia Antonina Kowerska als treue und wissenschaftlich orientierte Übersetzung konzipiert und kam ohne Illustrationen aus. Dahinter stand die Absicht, diese Märchen als Erbe der volkstümlichen Poesie unverfälscht wiederzugeben. Ihr war kein Erfolg beschieden und es kam zu keinen weiteren Auflagen.

In den weiteren Jahren kamen freie und wesentlich erfolgreichere Bearbeitungen auf den Markt. Viele davon stammten von Artur Oppmann. Zwischen 1918 und 1919 erschienen unter dem Namen Brüder Grimm mehr als 150 Auswahlbände. Hinzu kamen auch viele originär polnische Nacherzählungen, die dem Geschmack des Publikums noch stärker entsprachen als die Grimm-Ausgaben.

In der Zwischenkriegszeit waren die von Marceli Tarnowski stark abgeänderten, pädagogisierten und milderen Versionen, die als freie Bearbeitungen der ursprünglichen Märchen gelten können, bei Pädagogen und Eltern beliebt (vgl. Wozniak 2014: 50).

Mit den Schrecken des Zweiten Weltkrieges erlosch in Folge eine unvoreingenommene Rezeption der deutschen Märchen, die vielmehr als Nährboden für den Nationalsozialismus angesehen wurden 1 . Sie verschwanden für fast 10 Jahre völlig aus der polnischen Kinderliteratur.

${ }^{1}$ Während des Zweiten Weltkrieges wurden von der deutschen Besatzungsmacht bewilligte Übersetzungen (von Cecylia Niewiadomska, 1855-1925) herausgegeben. 
Ab dem Jahre 1956 etablierte sich in der Volksrepublik Polen dann ein Auswahlband von 22 Märchen mit kanonischer Geltung. Er (Auswahlband) war über Jahrzehnte hinweg Pflichtlektüre an den Schulen. Diese Märchen waren allerdings wieder bearbeitete und gekürzte Übersetzungen und entsprachen Tarnowskis Vorkriegsversionen (vgl. Pieciul-Karmińska 2013: 250). Eine 1982 erschienene zweite Gesamtausgabe, beruhend auf Tarnowskis Übersetzungen und weiteren von Emilia Bielicka, ist darüber hinaus zu erwähnen. Sie war allerdings weder besonders werktreu noch kommerziell erfolgreich. Aktuell gibt es zahllose Bearbeitungen und Nacherzählungen, die jedoch vorwiegend Motive der grimmschen Märchen aufgreifen und sich kaum an den Ursprungstexten orientieren.

Im Jahr 2010 unternahm der Media-Rodzina-Verlag mit werktreuen Übersetzungen der Germanistin Eliza Pieciul-Karmińska unter dem Titel Baśnie dla dzieci i dla domu (Märchen für Kinder und das Haus) den Versuch, dem polnischem Publikum erstmals das Kulturgut der Märchen in einer unverfälschten Sprache zu präsentieren.

\section{KINDERLITERARISCHES ÜBERSETZEN}

Für die Übersetzung von Märchen kann angenommen werden, was allgemein für Kinderliteratur gilt: Kinderliteratur ist durch ein asymmetrisches Verhältnis der betroffenen Kommunikationspartner und zweitens - was man als dessen Folge ansehen kann - durch ihre doppelte Systemzugehörigkeit zu Literatur und Pädagogik geprägt (vgl. O'Sullivan \& Rösler 2000: 231). Die Ungleichheit der Diskurspartner zeigt sich in typischen Kommunikationsstrukturen, in denen die Zielgruppe nicht als entscheidungsbefugter Akteur auftritt. Aus dieser schiefen Ausgangslage entwickelt sich die Verquickung von Literatur für Kinder mit primär pädagogischen Intentionen². Kinderbücher gelten, je nach den sich wandelnden kulturellen Werten und Normen, als Sozialisations- und Enkulturationsinstrumente (vgl. Ewers 2000: 7). Seit den 1950er Jahren findet ein Rückgang explizit belehrender Texte statt (vgl. Umlauf 2005: 158), allerdings sind die Erwartungen an Kinderliteratur umfassender und ambitionierter geworden: sie soll "wertvolle" Unterhaltung liefern, die Entwicklung von Fantasie und ästhetischem Empfinden fördern; Wissen vermitteln, Leselust und Lesefertigkeiten fördern und als Erziehungs- und Sozialisierungsinstrument soll sie das Kind mit gesellschaftlichen Rollen bekannt machen und seine eigene Mündigkeit entwickeln helfen.

\footnotetext{
${ }^{2}$ Diese Auffassung lässt sich bis in die Aufklärung zurückverfolgen, in der Kinderliteratur erstmals als eigenständige literarische Kategorie Kinder zu mündigen und urteilsfähigen Bürgern heranziehen sollte (vgl. Surmatz 2005: 28).
} 
Diese hohen Ansprüche an Kinderliteratur gehen allerdings einher mit einem geringen Ansehen, was sich in sämtlichen Bereichen von Produktion über Honorierung bis zu literaturwissenschaftlicher Forschung ausdrückt (vgl. Shavit 1986: 33).

Im Übersetzungsprozess wird das zuvor angesprochene asymmetrische Kommunikationsverhältnis noch vergrößert, indem Übersetzer, Lektorat und Verlage der Zielsprache ein weiteres Mal entscheiden, was dem kindlichen Leser zugemutet werden darf. Stolt sieht gar das Ausmaß der Abweichungen vom Ausgangstext als zentralen Unterschied zwischen Übersetzungen von Erwachsenenliteratur und Kinderliteratur (1978: 69)3.

Argumente dafür finden sich in der mangelhaften Weltkenntnis und Lebenserfahrung sowie der noch unvollständig entwickelten Sprachkompetenz und Rezeptionsfähigkeit des kindlichen Lesers (vgl. Reiß 1982: 8; O’Sullivan 1991: 5). Der Text wird bei der Übersetzung nachträglich meist „verbessert”. Diese Eingriffe, die beispielsweise die Erzählerrolle, den Ton oder typische zielkulturelle Erzählweisen umfassen, entspringen dem Kindheitsimage des Übersetzers (vgl. O'Sullivan 2000: 235 und 264, siehe auch „Superadressat" bei Oittinen 2000: 24). Ein Kindheitsimage enthält Werte und Normvorstellungen sowie überindividuelle Elemente, die gesamtgesellschaftliche Bedeutung haben. Dennoch bildet das Kindheitsimage vor allem eine Projektionsfläche, die Erfahrungen, Ideale, Wünsche, Ängste, usw. abbildet. Auch wenn Kinderliteratur vom ästhetischen Standpunkt betrachtet eine autonome, selbstständige Literatur darstellt, wird die Loyalität gegenüber dem Ausgangstext vielfältigen außerliterarischen Ansprüchen untergeordnet. Übersetzungstheoretisch ist diese Praxis durch die funktionale Skopostheorie legitimiert, die im Zieltext einen eigenständigen, vom Original unabhängigen Text sieht (vgl. Kaindl 1994: 116f.). Der Skopostheorie zufolge informiert eine "treue Übersetzung“ über die zielfunktionsrelevanten Merkmale des Ausgangstextes. Welche Merkmale als relevant gewertet und vermittelt werden, hängt von dem Übersetzungsauftrag und dem Zielrezipienten $a b$, auf deren Grundlage der Übersetzer Entscheidungen trifft.

Ein präskriptives Konzept der Äquivalenz ist zweifellos problematisch, weil jede Übersetzung zu Änderungen führt. Umso bezeichnender ist aber, dass sich der Stellenwert einer Literatur vor allem an einer sorgfältigen und wertschätzenden Übersetzung manifestiert (vgl. Osberghaus 1997: 12). Die Stellung des Ausgangstexts spielt also eine bedeutende Rolle für die Übersetzung 4 .

\footnotetext{
${ }^{3}$ Diese Feststellung hat nicht an Aktualität eingebüßt, wie folgendes Zitat zeigt: „[...] translation for children does not differ in kind from translating for adults, but simply in the extent to which it necessitates or allows forms of textual manipulation" (Van Coillie 2006: v).

${ }^{4}$ Surmatz (2005: 26f. und 383) weist das bei Klassikern der Erwachsenenliteratur nach, die mit dem Eingang in das kinderliterarische System unverzüglich einer Vielzahl von Eingriffen,
} 
Die Märchensammlung der Brüder Grimm gilt seit ihrer Ernennung als Weltdokumentenerbe im Juni 2005 als literarisches Denkmal - konkret betrifft das das Handexemplar der ersten Auflage von 1812. Es ist also anzunehmen, dass dieser offizielle Status dazu führt, dass die Märchen nicht mehr als bloße Vehikel zum Transfer pädagogischer und moralischer Inhalte betrachtet werden und bei ihrer Übersetzung eine größere Sorgfalt erfahren ${ }^{5}$. Am Beispiel des Textes Rotkäppchen werden im Weiteren zwei Umfragen dargestellt, in denen mehrere Studentengruppen zum Text befragt wurden bzw. Textpassagen übersetzen sollten.

\section{KRITIK AN ROTK̈̈PPCHEN}

In einer qualitativen Umfrage unter 17 Studierenden am Ende des Masterstudiums der angewandten Linguistik und Translatorik an der Universität Gdańsk im Wintersemester 2018 zeigte sich, dass das Märchen Rotkäppchen (Grimm \& Grimm 1857: 140ff.) relativ wenig Akzeptanz findet. Die überwiegende Mehrheit gab an, dass der Text für Kinder ungeeignet und zu grausam / vulgär sei, wogegen sich nur eine Person vorbehaltlos positiv äußerte. Etwa die Hälfte der Befragten gab an, dass das Märchen erst nach umfangreichen Veränderungen Kindern zugänglich gemacht werden solle.

Die häufigste Stellungnahme betraf die Frage des Wolfes: „Was trägst du unter der Schürze?" Die Doppeldeutigkeit der Frage stieß fast alle Befragten $a b$, auch unter dem Vorbehalt, dass Kinder eine sexuelle Anspielung vermutlich nicht verstehen würden. Als problematisch wurden auch vulgäre oder brutale Ausdrücke wie "Maul", „fressen“, "packen“ oder auch „ein paar Schnitte“ (mit der Schere) eingestuft. Das galt auch für die „Flasche Wein", die in einem Märchen nichts verloren habe. Selbst von der Minderheit, die dem Text grundsätzlich positiv gegenüberstand, wurde die Notwendigkeit betont, problematische Begriffe abzuändern, um Kinder nicht zu erschrecken. Als grausam bzw. aggressiv wurde auch der Schluss des Märchens, in welchem Rotkäppchen und die Großmutter den Wolf überlisten, empfunden. Er verneble laut den Befragten die Moral der Erzählung, deren zentrale Botschaft: „Man soll immer auf die Mutter hören“ laute.

Ein Viertel der Befragten lehnte zudem die Handlung (Aufschneiden des Bauches, Fressen der Großmutter und von Rotkäppchen) als veraltet ab. Ein

\footnotetext{
Kürzungen, Streichungen usw. ausgesetzt sind. Sie zeigt auch, dass mit zunehmendem Ansehen des Autors oder des Textes (z.B. bei Büchern von Astrid Lindgren) die Zahl der Textveränderungen abnimmt.

${ }^{5}$ Für polnische Übersetzungen forderte Maria Krysztofiak schon länger, diesen Mangel zu beheben (siehe Krysztofiak 1999).
} 
Zitat zeigt das sehr gut: „Es hat sich viel geändert in den Märchen. Jetzt ist es unmöglich, den Kindern Wein zu geben, sie alleine durch den Wald gehen zu lassen und mit Wölfen sprechen zu lassen".

Bei der Befragung wurde mehrmals auf Unterschiede zu geläufigen polnischen Versionen hingewiesen, in denen der Wolf etwa im Fluss ertrinkt oder vom Jäger erschossen wird. Die Studierenden erklärten ihre negativen Urteile über den Text mit Hinweisen auf den belehrenden Zweck und den Einfluss von Märchen sowie darauf, dass Kinder Verhalten nachahmen. Nur eine befragte Person gab an, als Kind die grimmschen Märchen authentisch gehört und auch gemocht zu haben. Sie äußerte auch die Überzeugung, dass Anspielungen und Gewalt in Märchen Kinder nicht erschrecken würden.

Insgesamt stieß das Märchen in seiner Originalfassung aber auf Ablehnung, was teilweise damit erklärt werden kann, dass freie Bearbeitungen der Motive den Befragten vertrauter sind und deshalb die ursprüngliche Fassung als fremd empfunden wird. Die geäußerten Erwartungen hinsichtlich der moralischen Lehre zeigen zudem eine enge Verknüpfung von Märchen mit vorrangig pädagogischen Funktionen.

Obwohl diese offene Befragung subjektive Einstellungen erhob und nicht repräsentativ ist, spiegelt das Ergebnis wesentliche Tendenzen wider, die in einer weiteren Erhebung mit anderen Studierendengruppen geprüft werden sollen.

\section{HYPOTHESENBILDUNG}

Im Folgenden werden die Ergebnisse einer experimentellen Übersetzungssituation dargestellt, bei der eine Gruppe von 19 Studierenden in 2 Gruppen von jeweils 10 und 9 Personen geteilt wurde. Beiden Gruppen wurde der Text Rotkäppchen vorgelegt. Der 10-köpfigen Gruppe wurde die Information gegeben, dass es sich dabei um ein Kunstwerk im Range eines Weltdokumentenerbes handelt, die andere Gruppe, bestehend aus 9 Studierenden, erhielt diese Information nicht, sondern nur allgemeine Informationen über die Autorenschaft und das Entstehungsjahr des Textes. Der Übersetzungsauftrag enthielt lediglich die Information, dass der Zielrezipient der Übersetzung Kinder sind. Im Vorfeld wurde die Hypothese aufgestellt, dass sich die Übersetzung umso treuer an dem Ausgangstext orientiert, je bedeutender dessen Rang ist. Das müsste also bedeuten, dass es in beiden Gruppen einen signifikanten Unterschied bei den Übersetzungsleistungen gibt. Die Gruppe mit der reinen Information über den Text müsste bei der Übersetzung freier vorgehen und auch persönliche Haltungen zu Märchen und Texten für Kinder einfließen lassen, während die andere Gruppe, der die 
Information über den Status des Textes vermittelt wurde, sich treuer an die Übersetzung machen müsste. Ob sich diese Hypothese bestätigen lässt, wird im Weiteren gezeigt. Die beiden Gruppen werden im Weiteren als Gruppe A, d.h. ihr wurden Informationen über den Text als Bestandteil des Weltdokumentenerbes gegeben, und als Gruppe B, sie erhielt lediglich die Information, dass es sich bei dem Text um ein bekanntes Märchen der Brüder Grimm handelt, bezeichnet.

Zur Überprüfung wurden mehrere Textstellen zur Übersetzung vorbereitet, die einige Stichwörter enthalten, die in der zuvor mit einer anderen Studierendengruppe durchgeführten Umfrage als besonders problematisch eingestuft wurden. Pieciul-Karmińska (2009/2010: 64) hat einige dieser Textpassagen diskutiert, da sie in den meisten polnischen Übersetzungen sehr stark verändert wurden. Zu diesen Veränderungen zählen ihrer Analyse zufolge exzessive Diminuierung und purifizierende Substitutionen im Vergleich zu den deutschsprachigen Ausgangstexten.

Als Hilfen konnten die Studierenden Lexika und Wörterbücher, je nach Wahl des Studierenden online oder in Papierform, heranziehen. Unter den genannten Bedingungen wurden den beiden Gruppen folgende Textpassagen zur Übersetzung vorgelegt:

1) Rotkäppchen versprach der Mutter recht gehorsam zu sein.

2) „Was trägst du unter der Schürze?" - „die Großmutter ist krank und schwach, da bring ich ihr Kuchen und Wein, gestern haben wir gebacken, da soll sie sich stärken."

3) „Ei Großmutter, was hast du für große Hände?“ - „dass ich dich besser packen kann."

4) „Aber Großmutter, was hast du für ein entsetzlich großes Maul?“ "dass ich dich besser fressen kann."

5) Da nahm er die Schere und schnitt ihm den Bauch auf, und wie er ein paar Schnitte getan, da sah er das rote Käppchen leuchten.

Im Folgenden soll gezeigt werden, welche Übersetzungstechniken auf lexikalischer Ebene bei potenziell problematischen Einheiten angewendet werden in Abhängigkeit davon, ob über den Status des Werkes als Weltkulturerbe informiert oder der Text lediglich als bekanntes Märchen präsentiert wurde.

\section{GEWÄHLTE ÜBERSETZUNGSVARIANTEN}

Bei der Übersetzung des 1. Satzes waren mehrere Elemente von Interesse. Eines davon bezieht sich auf die Übersetzung von „Mutter“. In vielen Übersetzungen ins Polnische findet sich die Form "mamusia“ (Diminutivform, vergleichbar mit Mutti oder Mami). In den untersuchten Übersetzungen 
findet sich dagegen stets die Normalform „mamie“ bzw. „mamy“ (Mutter), in einem Fall bei Gruppe A auch die sehr formelle Form "obiecał swojej matce“ (Das Wort "Mutter" hat im Polnischen zwei Entsprechungen: „mama” und "matka", wobei die zweite eher formell ist). Die häufige und erwartbare Diminutivform "mamusia“ kommt also in dieser Untersuchung überraschenderweise nicht vor. Die Wahl von Diminutiven bzw. Normalformen lässt sich im Rahmen der gewählten Übersetzungsvarianten als ein Modus erkennen, der sich an den Polen Verstärkung bzw. Abschwächung orientiert. Die zu analysierenden Sätze weisen diese Orientierung häufig auf.

Tabelle 1. Übersetzung Satz 1

\begin{tabular}{|l|c|c|c|}
\hline \multicolumn{1}{|c|}{ Übersetzung Satz 1 } & $\begin{array}{c}\text { Gruppe A } \\
\text { (Total 9) }\end{array}$ & $\begin{array}{c}\text { Gruppe B } \\
\text { (Total 10) }\end{array}$ & Total 19 \\
\hline $\begin{array}{l}\text { Czerwony Kapturek obiecał mamié }, ~ \\
\text { Rotkäppchen hat der Mutter versprochen, }\end{array}$ & 9 (davon 1 matce) & 7 & 16 \\
\hline $\begin{array}{l}\text { Czerwony Kapturek obiecała mamie, } \\
\text { Rotkäppchen hat der Mutter versprochen, }\end{array}$ & 0 & 3 & 3 \\
\hline $\begin{array}{l}\text { że będzie (być) (bardzo) grzeczny } \\
\text { dass es sehr gehorsam sein wird }\end{array}$ & 1 & 2 & 2 \\
\hline $\begin{array}{l}\text { że będzie (być) grzeczna } \\
\text { dass es gehorsam sein wird }\end{array}$ & 0 & 2 & 7 \\
\hline $\begin{array}{l}\text { że będzie (być) posłuszny } \\
\text { dass es gehorsam sein wird }\end{array}$ & 5 & 2 & 3 \\
\hline $\begin{array}{l}\text { że będzie (być) (naprawdę) posłuszna } \\
\text { dass es sicher gehorsam sein wird }\end{array}$ & 1 & 0 & 1 \\
\hline $\begin{array}{l}\text { że nie zboczy z drogi } \\
\text { dass es nicht vom Weg abkommt }\end{array}$ & 0 & 1 & 1 \\
\hline $\begin{array}{l}\text { posłuchać się mamy } \\
\text { auf die Mutter zu hören }\end{array}$ & 1 & 0 & 2 \\
\hline $\begin{array}{l}\text { że będzie na siebie uważać } \\
\text { auf sich aufzupassen }\end{array}$ & & 3 & 2 \\
\hline
\end{tabular}

In Satz 1 tritt allerdings noch ein anderes Phänomen auf, das unabhängig von der zuvor genannten Strategie auftritt, im Kontext unserer Studie aber relevant erscheint. Es wird im Folgenden skizziert. Im Präteritum ist eine Genus-Gleichheit erforderlich, dieses Prinzip spielt bei der Übersetzung eine Rolle, da die Bezeichnung "Czerwony Kapturek" maskulin ist, wiewohl es sich um ein Mädchen handelt, welches im Polnischen femininen Kasus hat.

${ }^{6}$ Die Übersetzungen der Antworten der Studierenden stammen von den Autorinnen des Beitrags. 
Es wurden darum unterschiedliche Personalendungen bei der Übersetzung von Satz 1 „Rotkäppchen versprach der Mutter recht gehorsam zu sein.“ gewählt. Die Tabelle zeigt die gewählten Formen.

Die gesamte Gruppe A, die Informationen über den Status des Werkes als Weltkulturerbe erhalten hatte, wählte die männliche Form des ersten finiten Verbs, d.h. obiecał. Freier ging die Gruppe B vor, die in 3 Fällen eine weibliche Form wählte. Aus der Tabelle kann man außerdem erkennen, dass es zu Inkongruenz kam: ein männliches Verb im Präteritum mit einem Adjektiv mit femininer Endung. Das war jeweils einmal der Fall in Gruppe A und B. In jeweils 1 Fall in beiden Gruppen wurden Infinitivkonstruktionen gewählt.

Die Übersetzung der Elemente „recht gehorsam zu sein“ variiert, darunter findet sich die sehr freie Variante "że nie zboczy z drogi“ (vom Weg abkommen) (1 Fall, Gruppe A).

In keiner der Übersetzungen findet sich eine Diminutivform. Das Adverb „recht" wurde selten übersetzt, nur in der Gruppe A gab es dazu 2 Beispiele (bardzo, naprawdę). Sie sind in Klammern angefügt und werden nicht separat aufgelistet.

Eine weitere Problematik zeigt die Übersetzung von Satz 2: „Was trägst du unter der Schürze?" - "die Großmutter ist krank und schwach, da bring ich ihr Kuchen und Wein, gestern haben wir gebacken, da soll sie sich stärken." In der nachfolgenden Tabelle wird nicht auf die gesamte Passage, sondern nur auf das bereits genannte problematische Element „unter der Schür$\mathrm{ze}^{\prime \prime}$ eingegangen.

Tabelle 2. Übersetzung Satz 2

\begin{tabular}{|l|c|c|c|}
\hline Übersetzung Satz 2 „unter der Schürze“ $^{\text {„un }}$ & $\begin{array}{c}\text { Gruppe A } \\
\text { (Total 9) }\end{array}$ & $\begin{array}{c}\text { Gruppe B } \\
\text { (Total 10) }\end{array}$ & Total 19 \\
\hline $\begin{array}{l}\text { pod fartuszkiem } \\
\text { unter dem Schürzchen }\end{array}$ & 5 & 3 & 8 \\
\hline $\begin{array}{l}\text { w (swoim) fartuszku } \\
\text { in dem (seinem) Schürzchen }\end{array}$ & 4 & 0 & 4 \\
\hline $\begin{array}{l}\text { pod fartuchem } \\
\text { unter der Schürze }\end{array}$ & 0 & 2 & 2 \\
\hline $\begin{array}{l}\text { w (swoim) koszyczku } \\
\text { in seinem Körbchen }\end{array}$ & 0 & 4 & 4 \\
\hline $\begin{array}{l}\text { w koszyku } \\
\text { im Korb }\end{array}$ & 0 & 1 & 1 \\
\hline
\end{tabular}

Alle Formen der Übersetzung von "Schürze“ in der Gruppe A sind Diminutive, sie fallen also unter die Strategie der Abschwächung. Die Gruppe B wählte dagegen in 2 Fällen eine nicht diminuierte Form: „fartuch“ (Schürze), 
darüber hinaus finden sich auch 5 Fälle, in denen das Lexem frei als "koszyk" (Korb, in 1 Fall) oder in der Verkleinerung als „koszyczek“ (Körbchen, in 4 Fällen) ins Polnische übertragen wurde. In Kenntnis der zahlreichen polnischen Übersetzungen mögen die Normalformen "fartuch" (Schürze) sowie "koszyk“ (Korb) aus der Perspektive der Probanden unnatürlich für ein Märchen klingen. Die Verwendung dieser Formen ist jedenfalls dem Versuch geschuldet, nahe dem Ausgangstext zu übersetzen.

Es stand jeder Gruppe die Möglichkeit offen, zu der Übersetzungsübung eigene Notizen anzugeben. Im Zusammenhang mit der Übersetzung der Phrase "unter der Schürze“ kamen von Gruppe A 3 Anmerkungen zu Problemen mit der Übersetzung der Präposition, wobei angegeben wurde, dass „pod fartuszkiem“ (unter dem Schürzchen) auf Polnisch seltsam klinge. Diese explizierte Überlegung weist darauf hin, dass die Gruppe A mit dem Kenntnishintergrund, dass es sich bei dem Text um ein Weltkulturerbe handelt, den Versuch unternahm, sich von den bekannten Übersetzungen eher $\mathrm{zu}$ distanzieren und textnäher zu arbeiten. Allerdings wurde in Gruppe B ebenfalls angemerkt, dass die Kenntnis polnischer Übersetzungen die vorgelegte Arbeit beeinflusst und auch erschwert habe.

Bei der Übersetzung von Satz 3: „Ei Großmutter, was hast du für große Hände?" - "dass ich dich besser packen kann.", werden im Folgenden die Elemente „Hände" sowie „das Verb „packen“ analysiert.

Tabelle 3. Übersetzung Satz 3

\begin{tabular}{|l|c|c|c|}
\hline \multicolumn{1}{|c|}{ Übersetzung Satz 3 „Hände“ } & Gruppe A (Total 9) & Gruppe B (Total 10) & Total 19 \\
\hline $\begin{array}{l}\text { ręce } \\
\text { Arme/Hände }\end{array}$ & 8 & 7 & 15 \\
\hline $\begin{array}{l}\text { dłonie } \\
\text { Hände }\end{array}$ & 1 & 3 & 4 \\
\hline
\end{tabular}

Bei der Übersetzung der Phrase: „große Hände“ verwendete die Mehrheit der Probanden "ręka" (Arm/Hand), in 4 Fällen (1 Fall in Gruppe A und 3 Fälle in Gruppe B) verwendeten dagegen "dłon" (Hand). "Ręka" (Arm/Hand) ist breiter in der Bedeutung, das Wort bezeichnet im Alltagsgebrauch sowohl den ganzen Arm als auch die Hand, sowie üblicher in seiner Verwendung. „Dłoń“ (Hand) dagegen hat eine engere Bedeutung und gehört einem gehobenen Sprachregister an. Daraus lässt sich schließen, dass Gruppe B mit „dłon" häufiger eine freie Variante der Übersetzung gewählt hatte, die in unserer Analyse anhand der Orientierungspole Verstärkung und Abschwächung als Abschwächung bzw. Abmilderung verortet werden kann. 
Tabelle 4. Übersetzung Satz 3, 2. Element

\begin{tabular}{|l|c|c|c|}
\hline \multicolumn{1}{|c|}{ Übersetzung Satz 3 „packen“ } & Gruppe A (Total 9) & Gruppe B (Total 10) & Total 19 \\
\hline $\begin{array}{l}\text { lepiej chwycić } \\
\text { besser ergreifen }\end{array}$ & 2 & 0 & 2 \\
\hline $\begin{array}{l}\text { lepiej / mocniej / dobrze / łatwiej objąć } \\
\text { besser / stärker / leichter umarmen }\end{array}$ & 5 & 5 & 10 \\
\hline $\begin{array}{l}\text { lepiej przytulić } \\
\text { besser umarmen / kuscheln }\end{array}$ & 0 & 1 & 1 \\
\hline $\begin{array}{l}\text { lepiej złapać } \\
\text { besser erwischen / fangen }\end{array}$ & 0 & 0 & 4 \\
\hline $\begin{array}{l}\text { lepiej trzymać } \\
\text { besser halten }\end{array}$ & 1 & 0 & 1 \\
\hline $\begin{array}{l}\text { mocniej uścisnać } \\
\text { stärker umarmen }\end{array}$ & 1 & 4 & 1 \\
\hline
\end{tabular}

Als weiteres Element in Satz 3 wird die Übersetzung des Verbs „packen“ analysiert.

Das Aktionsverb "packen" wird mit den Aktionsverben "chwycić", "objąćc", "przytulićc", "złapać" und "uścisnąćc übersetzt. Es gibt auch ein Zustandsverb: "trzymać" (halten, 1 Fall in A). Von den Aktionsverben sind 3 mit positiver bzw. eher positiver Konnotation: "objąć" (umarmen, jeweils 5 in A und B), "przytulić" (umarmen, kuscheln 1 B), "uścisnąćc (umarmen, 1 in A) und 2 mit neutraler oder negativer Konnotation: „złapać” (erwischen, fangen 4 in B) und "chwycić" (ergreifen, 2 in A).

Es zeigt sich also, dass die Mehrheit beider Gruppen mit dem Verb „objąć (umarmen) eine Lösung gewählt hat, die eine abschwächende Funktion hat.

Tabelle 5. Übersetzung Satz 4

\begin{tabular}{|l|c|c|c|}
\hline \multicolumn{1}{|c|}{ Übersetzung Satz 4 „Maul“ } & Gruppe A (Total 9) & Gruppe B (Total 10) & Total 19 \\
\hline $\begin{array}{l}\text { takie (przerażająco) wielkie zęby } \\
\text { so entsetzlich große Zähne }\end{array}$ & 6 & 1 & 7 \\
\hline $\begin{array}{l}\text { tak (strasznie / taką straszliwie) wie- } \\
\text { lką / dużą / ogromną buzię } \\
\text { so ein entsetzlich großes Gesicht }\end{array}$ & 2 & 4 & 6 \\
\hline $\begin{array}{l}\text { taki przerażąjący pysk } \\
\text { so eine schreckliche Schnauze / ein } \\
\text { schreckliches Maul }\end{array}$ & 1 & 0 & 1 \\
\hline $\begin{array}{l}\text { taką wielką / dużą paszczę } \\
\text { so eine große Schnauze/ein großes Maul }\end{array}$ & 0 & 3 & 3 \\
\hline $\begin{array}{l}\text { taka przerażająca / strasznie duża twarz } \\
\text { so ein schrecklich großes Gesicht }\end{array}$ & 0 & 2 & 2 \\
\hline
\end{tabular}


Nur 6 von 19 Fällen in beiden Gruppen insgesamt weisen ein negativ konnotiertes Verb auf und vermitteln eine verstärkende Funktion. Die Unterschiede zwischen der Gruppe A und B sind allerdings nicht erheblich.

Die Analyse der Übersetzung des 4. Satzes fokussiert auf die Elemente "(entsetzlich großes) Maul" sowie "fressen".

Dem Inhalt entsprechend wurden die Formen "paszcza“ (Schnauze) bzw. „pysk“ (Maul) verwendet. In 7 Fällen lautet die Übersetzung „zęby“ (Zähne), eine Form, die den Studierenden in ihnen bekannten Versionen des Märchens begegnet ist und die sie in der Übersetzung auf der Ebene einer semantischen Anpassung sicherlich beeinflusst hat. Aus inhaltlicher Perspektive ist "paszcza“ (Schnauze) weniger als "pysk“ (Maul) passend für das Nomen Wolf. Die Formen "twarz" (Gesicht, wobei hier allerdings ein direkter Bezug auf die Großmutter und nicht den Wolf hergestellt wird) und "buzia" (umgangssprachlich und niedlich für Gesicht) kommen auch in vielen populären Übersetzungen vor und können als vertraute Varianten erachtet werden. Für "buzia“ lässt sich festhalten, dass es eher aus der gesprochenen Sprache stammt und weniger formell ist. Generell erscheinen die Lexeme „buzia“ und "twarz" aber im Verhältnis zum Ausgangstext als fehlerhaft und sind somit Beispiele für äußerst freie Übersetzungen.

In Zusammenhang mit den Übersetzungsvarianten, die dem Modus Verstärkung bzw. Abschwächung unterliegen, lassen sich die Formen: „zęby“ (Zähne), "pysk“ (Maul), „paszcza“ (Schnauze) einer eher angsteinjagenden Funktion zuordnen. Dagegen dient die Form „buzia“, die im Polnischen sehr niedlich und kindlich ist, vor allem der Abschwächung. Gewöhnlich verwendet man sie mit Kindern und in der Kollokation "ładna buzia" (ein schönes Gesicht). Die gewählten Übersetzungen "straszliwie ogromna/wielka/duża buzia" erweisen sich demnach als etwas widersprüchlich (ein entsetzlich großes (schönes) Gesicht). Mit "twarz" wurde eine Form mit neutraler Konnotation gewählt, die allerdings ein Körperteil bezeichnet und in dem Kontext nicht stimmig ist.

Zusammenfassend tendiert Gruppe A dazu, in der Übersetzung den Kontext nicht abzumildern, das zeigen die 6 Fälle, in denen "zęby“ und 1 Fall, in dem "pysk“ gewählt wurden. In der Gruppe B variieren die Übersetzungsvarianten allerdings stärker. Die Form „zęby“ kann insgesamt als gebräuchlichste Variante in den existierenden polnischen Rotkäppchen-Übersetzungen betrachtet werden.

Der 2. Teil des 4. Satzes interessiert im Rahmen der Analyse vor allem durch die Übersetzung des Verbs "fressen“.

Bei den in der Übersetzung verwendeten Verben finden sich: „pożreć" (verschlingen, fressen - auch beim Menschen, hastig bzw. sehr viel essen), "zjeść" (essen, verzehren), „schrupać“ (knuspern, knabbern), „zeżreć" (fressen, pejorativ für Menschen). 
Tabelle 6. Übersetzung Satz 4, 2. Element

\begin{tabular}{|l|c|c|c|}
\hline \multicolumn{1}{|c|}{ Übersetzung Satz 4 „fressen“ } & Gruppe A (Total 8) & Gruppe B (Total 10) & Total 18 \\
\hline $\begin{array}{l}\text { łatwiej / lepiej zjeść / żeby móc cię zjeść } \\
\text { damit ich dich besser essen kann }\end{array}$ & 7 & 4 & 11 \\
\hline $\begin{array}{l}\text { lepiej było mi cię pożreć } \\
\text { damit ich dich besser fressen kann }\end{array}$ & 0 & 5 & 5 \\
\hline $\begin{array}{l}\text { lepiej schrupać } \\
\text { besser knabbern }\end{array}$ & 0 & 1 & 1 \\
\hline $\begin{array}{l}\text { lepiej zeżreć } \\
\text { besser fressen }\end{array}$ & 1 & 0 & 1 \\
\hline
\end{tabular}

Es zeigt sich, dass nur 1 Proband in Gruppe A das Verb „zeżreć“ (fressen, pejorativ für Menschen) verwendet, was aber semantisch nicht stimmig ist (vgl. „pożreć" - fressen - als passendere Variante), 7 Fälle verwenden mit "zjeść" (essen) ein neutrales Verb, das in diesem Kontext aber als Abschwächung des Inhalts zu klassifizieren ist. In Gruppe A konnte ein Proband keine Übersetzung dieser Phrase abliefern. Gruppe B dagegen verwendet zur Hälfte mit „pożreć" (fressen) ein Verb, das nicht abschwächend wirkt, sondern ziemlich genau dem Ausgangstext entspricht. In 1 Fall findet sich das Verb "schrupać" (essen im Sinne von knabbern), welches semantisch nicht dem Ausgangstext entspricht und aufgrund seiner Bedeutung als Abschwächung zu deuten ist. Bei den beiden Gruppen lässt sich feststellen, dass Gruppe A sehr stark zu einer abschwächenden Übersetzung tendiert, während Gruppe B stärker variiert und auch den Inhalt eher beachtet.

Bei dem Satz 5 ist das zu analysierende Element die Übersetzung von "Schere".

Tabelle 7. Übersetzung Satz 5

\begin{tabular}{|l|c|c|c|}
\hline \multicolumn{1}{|c|}{ Übersetzung Satz 5 „Schere“ } & Gruppe A (Total 9) & Gruppe B (Total 10) & Total 19 \\
\hline $\begin{array}{l}\text { Wtedy wziął wielkie nożyce. } \\
\text { Dann nahm er eine sehr große Schere. }\end{array}$ & 1 & 0 & 1 \\
\hline $\begin{array}{l}\text { Wtedy wziął nożyczki. } \\
\text { Dann nahm er eine (kleine) Schere. }\end{array}$ & 5 & 5 & 10 \\
\hline $\begin{array}{l}\text { Myśliwy wziął nożyce. } \\
\text { Der Jäger nahm eine große Schere. }\end{array}$ & 0 & 4 & 1 \\
\hline $\begin{array}{l}\text { Wtedy wziął nożyce. } \\
\text { Dann nahm er eine große Schere. }\end{array}$ & 2 & 0 & 6 \\
\hline $\begin{array}{l}\text { Wyciągnął nożyczki. } \\
\text { Dann nahm er eine (kleine) Schere. }\end{array}$ & 1 & 1 & 1 \\
\hline
\end{tabular}


Bei der Übersetzung zeigen sich für "Schere“ die beiden Formen "nożyczki“ (kleine Schere, sehr oft im Gebrauch) und "nożyce" (eine große Schere). Es zeigen sich hier wiederum verstärkende (v.a. das Beispiel „wielkie nożyce" - eine sehr große Schere) und abschwächende Strategien mit der Entsprechung "nożyczki“ (eine kleine Schere). Gruppe A hat in 3 Fällen eine verstärkende Form und in 5 Fällen eine gemilderte Diminutivform gewählt, Gruppe B hat die Formen jeweils zur Hälfte angewendet. Interessant ist auch ein Fall bei Gruppe B, in dem das Subjekt „myśliwy“ (Jäger) explizit genannt wird.

\section{ZUSAMMENFASSUNG}

Die dieser vorliegenden Arbeit zugrunde liegende Hypothese, dass nämlich der konkrete Text Rotkäppchen durch die Kenntnis, dass er als Weltdokumentenerbe einen hohen literarischen Status aufweist, adäquater und treuer übersetzt werde konnte durch die durchgeführte Untersuchung nicht bestätigt werden. Gruppe A und B unterscheiden sich kaum darin, wie treu sie der Textvorlage begegnen. In einigen Fällen variieren die lexikalischen Elemente bei Gruppe B stärker. Gruppe A lieferte allerdings keine loyaleren Übersetzungen als Gruppe B.

Es ließen sich aber einige Beobachtungen anstellen, die durchaus relevant sind. So haben die Probanden auf die in Vorfeld erhobenen Elemente besonders sensibel reagiert, was sich in Übersetzungsleistungen zeigt, die eine abschwächende Funktion aufweisen. Diese Ergebnisse liegen auf lexikalischer Ebene vor und sie verweisen darauf, dass nicht etwa die Information über den Status des Textes ausschlaggebend für die gewählte Übersetzungsvariante ist, sondern vielmehr die nicht erhobene und zudem systematisch kaum erhebbare Beeinflussung eigener Leseerfahrung. Man kann davon ausgehen, dass alle Probanden den Text Rotkäppchen als Kinder kennen gelernt haben, allerdings in vielfältigen Adaptionen und freien Nacherzählungen. Anhaltspunkte dafür, dass dies die Übersetzungsleistung nachhaltig beeinflusst, gibt es mehrere. Auf der lexikalischen Ebene finden sich Elemente wie, wie etwa das Wort "zęby“ (Zähne), das in den bekannten polnischen Rotkäppchen-Übersetzungen als übliche Variante vorherrscht, bzw. ist auf das Lexem "koszyczek“ (Körbchen) oder "koszyk“ (Korb) hinzuweisen, das in dem Ausgangstext (der zu übersetzenden Phrase) überhaupt keine Entsprechung hat (,Schürze"). In diesen Kontext fällt auch die Erweiterung durch "myśliwy" (Jäger).

Ein auffälliger Modus, der den überwiegenden Teil der Übersetzungsarbeiten durchzieht, ist die Abschwächung (im Gegensatz zur Verstärkung). 
Diese Beobachtung steht auch damit in Einklang, dass die im polnischen Sprachraum bekannten Märchenübersetzungen der Brüder Grimm zumeist geschönt und idealisiert sind. Unsere Untersuchung konnte die angenommene Hypothese nicht bestätigen, aber sie zeigt deutlich, dass die Übersetzungsvarianten eine Fortführung der eigenen Erfahrungen mit Märchen sind, die den Übersetzungsprozess wesentlich prägen.

\section{LITERATURVERZEICHNIS}

Ewers, H.-H. (2000). Was ist Kinder- und Jugendliteratur? Ein Beitrag zu ihrer Definition und zur Terminologie ihrer wissenschaftlichen Beschreibung. In: G. Lange (Hrsg.), Taschenbuch der Kinder- und Jugendliteratur (S. 2-16). Hohengehren: Schneider.

Grimm, J. / Grimm, W. (1857). Rothkäppchen. In: J. Grimm / W. Grimm (Hrsg.), Kinder- und Haus-Märchen. Bd. 1. Große Ausgabe (S. 140-144). Göttingen: Dieterich.

Grimm, J. / Grimm, W. (1896). Bajki domowe i dziecinne (übersetzt von Zofia Antonina Kowerska). Warszawa: Księgarnia M. Arcta.

Grimm J. / Grimm, W. (1956). Baśnie (übersetzt von Marceli Tarnowski). Warszawa: Nasza Księgarnia.

Grimm, J. / Grimm, W. (1982). Baśnie braci Grimm: Baśnie domowe i dziecięce zebrane przez braci Grimm (übersetzt von Marceli Tarnowski und Emilia Bielicka). Warszawa: Ludowa Spółdzielnia Wydawnicza.

Grimm, J. / Grimm W. (1989). Baśnie braci Grimm (übersetzt von Marceli Tarnowski und Emilia Bielicka). Warszawa: Ludowa Spółdzielnia Wydawnicza.

Grimm, J. / Grimm, W. (2008). Baśnie (übersetzt von Cecylia Niewiadomska). Kraków: Greg.

Grimm, J. / Grimm, W. (2010). Baśnie dla dzieci i dla domu (übersetzt von Eliza Pieciul-Karmińska). Poznań: Media Rodzina.

Halub, M. (1986). Die Märchen der Brüder Grimm in Polen. In: L. Denecke (Hrsg.), Brüder Grimm Gedenken 6 (S. 215-240). Marburg: E. N. Elwert.

Kaindl, K. (1994). ,Let's have a party!' Übersetzungskritik ohne Original? Am Beispiel der Bühnenübersetzung. In: M. Snell-Hornby / F. Pöchhacker / K. Kaindl (Hrsg.), Translation studies - An interdiscipline (S. 115-126). Amsterdam: John Benjamins Publishing.

Krysztofiak, M. (1999). Przekład literacki a translatologia. Poznań: Wydawnictwo Naukowe UAM.

Oittinen, R. (2000). Translating for children. New York: Garland Publishing.

Osberghaus, M. (1997). Die Zeitgebundenheit kinderliterarischer Übersetzungspraxis. Analyse eines exemplarischen Falles. Magisterarbeit. Frankfurt a. M.: J. W. Goethe Universität.

O'Sullivan, E. (1991). Bausteine für ein Seminar zum kinderliterarischen Übersetzen. Mitteilungen des Instituts für Jugendbuchforschung, 2, 4-10.

O'Sullivan, E. (2000). Kinderliterarische Komparatistik. Heidelberg: C. Winter.

O’Sullivan, E. / Rösler, D. (2000). Wenn aus der Mad Tea Party ein deutsches Kaffeekränzchen wird. Zielkulturelle Adaptionen in Übersetzungen von Kinderliteratur als Mittel zur Bewußtmachung interkulturellen Transfers in der Lehrerbildung. In: L. Redella (Hrsg.), Wie ist Fremdverstehen lehr- und lernbar? (S. 231-253). Tübingen: Narr Francke Attempto.

Pieciul-Karmińska, E. (2009/2010). A Polish history of the Grimm fairy tales. Przektadaniec. A Journal of Literary Translation, 22-23, 57-75. 
Pieciul-Karmińska, E. (2013). Wer hat Angst vor den Brüdern Grimm? Zur Geschichte und Gegenwart der Kinder- und Hausmärchen in Polen. In: C. M. Pecher (Hrsg.), Märchen (k)ein romantischer Mythos? Zur Poetologie und Komparatistik von Märchen (S. 249-267). Hohengehren: Schneider.

Reiß, K. (1982). Zur Übersetzung von Kinder- und Jugendbüchern. Theorie und Praxis. Lebende Sprachen, 27/1, 7-13.

Shavit, Z. (1986). Poetics of children's literature. Athen / London: University of Georgia Press.

Stolt, B. (1978). How Emil Becomes Michel. On the Translation of Children's Books. In: G. Lathey (2006) (Hrsg.), The translation of children's literature. A reader (S. 67-83). Buffalo: Multilingual Matters Ltd.

Surmatz, A. (2005). Pippi Langstrumpf als Paradigma. Die deutsche Rezeption Astrid Lindgrens und ihr internationaler Kontext. Tübingen: Francke.

Umlauf, K. (2005). Moderne Buchkunde. Bücher in Bibliotheken und im Buchhandel heute. Wiesbaden: Otto Harrassowitz.

Van Coillie, J. (2006). Editors' preface. In: J. Van Coillie / W. P. Verschueren (Hrsg.), Children's literature in translation. Challenges and strategies (S. V-IX). Manchester: St. Jerome Publishing.

Wozniak, M. (2014). Die Kinder- und Hausmärchen in Poland. In: V. Joosen / G. Lathey (Hrsg.), Grimms' tales around the globe: The dynamics of their international reception (S. 39-58). Detroit: Wayne State University Press.

Received: 11.12.2018; revised: 5.09.2019 